\title{
Study on the relationship between liver damage caused by anti-tuberculosis drug rifampicin and related metabolic enzyme gene polymorphisms
}

\section{Qiang Su}

Nanchong Central Hospital, North Sichuan Medical College

Qiao Liu

Nanchong Central Hospital, North Sichuan Medical College

Juan Liu

Nanchong Central Hospital, North Sichuan Medical College

Lingyun $\mathrm{Fu}$

Nanchong Central Hospital, North Sichuan Medical College

Tao Liu

Nanchong Central Hospital, North Sichuan Medical College

Jing Liang

Nanchong Central Hospital, North Sichuan Medical College

Hong Peng

Nanchong Central Hospital, North Sichuan Medical College

Xue Pan ( $\sim$ pan198400@126.com)

The First Affiliated Hospital of Chongqing Medical University

\section{Research Article}

Keywords: Tuberculosis, liver damage, rifampicin, gene polymorphisms

Posted Date: July 14th, 2021

DOI: https://doi.org/10.21203/rs.3.rs-606273/v1

License: (c) (1) This work is licensed under a Creative Commons Attribution 4.0 International License. Read Full License 


\section{Abstract \\ Background}

The occurrence of Anti-TB Drug-induced Liver Injury (ATLI) affects the progress of anti-tuberculosis treatment and the cure rate of tuberculosis. To understand the mechanism and risk factors of ATLI, it is helpful to reduce or avoid the occurrence of liver damage. It is of great significance for the smooth completion of anti-tuberculosis treatment.

\section{Methods}

In this study, 2255 pulmonary tuberculosis patients were included as the research object, and their medical records were reviewed, questionnaire surveys, liver function tests at the end of February (including patients with uncomfortable symptoms during the intensive treatment period) and blood samples were saved. Determine cases of liver damage based on the criteria for liver damage. The genotype was determined by the PCR-LDR method, and the single factor and unconditional Logistic regression methods were used to explore the relationship between genetic polymorphism and ATLI susceptibility.

\section{Results}

Through the screening of related factors affecting ATLI, this study found that ATLI may be related to gender, year and age is related to body weight; there is an association between the genetic polymorphism site CYP2C9 *2 and ATLI, and the best association model is a dominant inheritance model.

\section{Conclusions}

In-depth discussion of the genetic nature of ATLI is of great practical significance and practicality for preventing the occurrence of liver damage in patients with tuberculosis in our region, reducing the damage to the liver by anti-tuberculosis drugs, and formulating individualized chemotherapy regimens to avoid treatment failure due to liver damage.

\section{Introduction}

Tuberculosis (TB) is a chronic infectious disease mainly transmitted by respiratory tract, which has seriously harmed human health for thousands of years. So far, it is still a major global public health and social problem[1]. In view of the severe situation of global tuberculosis and the experience of tuberculosis control in the world, since 1995, under the advocacy of who and Stop TB Partnership, with the joint efforts of international anti tuberculosis and lung disease alliance and other organizations and governments, countries and regions with high burden of tuberculosis have fully implemented direct 
chemotherapy Observed Treatment. Short Course (dots) as the core of the modern tuberculosis control strategy, its main contents include: the government's political commitment to the national tuberculosis control program; the detection of infectious tuberculosis through sputum smear examination as the main means of finding patients; under the direct observation and supervision, the patients are given free, standard short-term chemotherapy treatment; regular and uninterrupted supply of anti tuberculosis drugs as a guarantee It is an important measure for the success of DOTS strategy to implement unified bidding and purchase drugs, and to establish and maintain a monitoring system for tuberculosis control program[2].

The core treatment of DOTS strategy is standard short-term chemotherapy, which is based on the reasonable combination of sterilization, bacteriostasis and prevention of drug resistance[3]. Three firstline drugs, INH, RIF and PZA, which are recommended by who as standard short-term chemotherapy regimens, can cause adverse drug reactions (ADRs) of different degrees and frequencies while killing tuberculosis[4]. The main adverse reactions are gastrointestinal reaction, liver damage, allergic reaction, auditory nerve damage, optic nerve damage, kidney damage, etc. the most common adverse reaction is gastrointestinal reaction, and the more serious and frequent adverse reaction is liver damage, and the resulting deaths have also been reported. The incidence of liver damage caused by antituberculosis drugs varies from $2.5 \%$ to $34.9 \%$ [5]. The reasons for the different incidence of liver damage caused by antituberculosis drugs in different countries and regions may be related to different treatment regimens, different criteria for judging whether or not the liver damage caused by antituberculosis drugs occurs and its severity, and demographic differences. According to many studies, the occurrence of liver damage caused by antituberculosis drugs not only affects the medication compliance of patients, but also increases the risk of multidrug resistance due to irregular medication[6]. For example, the delay of treatment or aggravation of the disease due to drug withdrawal may lead to the failure of short-term chemotherapy and even death of patients, which has a negative impact on the implementation of tuberculosis prevention and control plan in China[7]. Therefore, it is of great significance to understand the mechanism and predisposing factors of liver damage caused by antituberculosis drugs, so as to reduce or avoid the occurrence of liver damage and complete the antituberculosis treatment smoothly.

The mechanism of antituberculosis drug-induced liver injury (Atli) has not been fully elucidated. At present, studies suggest that isoniazid (INH, $H)$, rifampicin (RIF, R) and pyrazinamide (PZA, $z$ ) are the main antituberculosis drugs with potential hepatotoxicity[8]. These enzymes are involved in the production and metabolic excretion of toxic intermediates. Therefore, the activities of metabolic enzymes such as NAT2, CYP and GST may play an important role in the occurrence of Atli. In recent years, the association between genetic polymorphisms of the genes encoding these drug metabolizing enzymes (DMEs) and Atli has become a hot topic in pharmacogenomics in the post genomic era.

The objective of this study is to analyze the relationship between Atli susceptibility and gene polymorphism from the perspective of genetics, reveal the genetic characteristics of Atli and find out the drug metabolism enzyme genes with predictive value by genotyping CYP3A4 enzyme, CYP2C9 enzyme and CYP2C19 enzyme. 


\section{Materials And Instruments}

Agrose LE: Shanghai Gene Technology Co., Ltd.; PCR system: Qiagen hotstar Taq enzyme system (enzyme $5 \mu / \mu l$, buffer, Q-solution, Mg2+); Axygene PCR microplate 96 well; Axygene PCR tubes $(0.2 \mathrm{ml})$; LDR System: NEB Taq DNA ligase enzyme system (enzyme $5 \mu / \mu$ l, buffer); whole blood genomic DNA extraction kit: Tiangen Biochemical Technology Co., Ltd. DP319 kit. Pipette: Eppendorf Company; Centrifuge: American sigma; HHS electronic constant temperature water bath: Shanghai Boxun Industrial Company; Automatic microplate reader: BIO-RAD; Water Purifier: French MILLPORE; LSZM-60KCS vertical

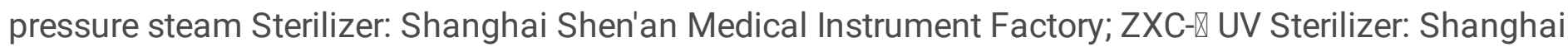
Yuejin Medical Optical Instrument Factory; MOV-313P Dry Heat Sterilizer: Japan SANYO; Digital Display Blast Dryer: Shanghai Boxun Laboratory Co., Ltd. Medical equipment factory; Nucleic acid quantifier: BIORAD. Thermal cycler: PERKIN ELMER Gene Amp PCR system 9600; MJ PTC-200 Gradient cycler. Sequencer: ABI PRISM 377 DNA Sequencer; ABI PRISM 3730 DNA Sequencer. Electrophoresis system: Electrophoresis instrument JY600+: Beijing Junyi Dongfang Electrophoresis Equipment Co., Ltd.; FR-200A automatic ultraviolet and visible analysis device: Shanghai Furi Technology Co., Ltd.; Bioelectrophoresis image analysis system: Shanghai Furi Technology Co., Ltd.;

\section{Methods}

\section{Research object}

2560 patients with pulmonary tuberculosis were included in the study. According to the criteria of Atli, 251 patients with abnormal liver function were excluded. A total of 144 patients were included in the Atli case group, and 2255 patients with normal liver function were included in the control group.

Inclusion criteria: the diagnosis of pulmonary tuberculosis was in accordance with the standard of "guidelines for diagnosis and treatment of pulmonary tuberculosis" formulated by tuberculosis branch of Chinese Medical Association in 2001; patients with newly diagnosed and retreated pulmonary tuberculosis were accepted; free treatment agreement of China tuberculosis control program was signed and accepted for 6 months or more Nine months of free chemotherapy; voluntary participation in the survey and signing informed consent; liver function was completely normal before anti tuberculosis treatment (alt, AST, TBIL).

Exclusion criteria: those who did not meet the national conditions for free treatment; those who had mental or disability diseases and could not accept the survey; those who were unwilling to participate in the study for some reasons.

\section{DNA extraction from human peripheral blood}

The blood samples of the above subjects stored at $-20^{\circ} \mathrm{C}$ or below were dissolved at room temperature, and genomic DNA was extracted from $1 \mathrm{ml}$ venous blood according to the instructions using the whole blood genomic DNA extraction kit. Whole blood genomic DNA. The extraction process is as follows: $1 \mathrm{ml}$ 
of anticoagulant containing blood sample dissolved at room temperature is added with equal volume of cell lysate $\mathrm{Cl}$, and then reversed and mixed for 5 times, centrifuged at $8000 \mathrm{rpm}$ for 2 minutes, and the supernatant is discarded. Then add $1.5 \mathrm{ml}$ cell lysate $\mathrm{CL}$ to it, mix it upside down for 5 times, centrifuge at 8000rpm for 2 minutes, discard the supernatant, and place the centrifuge tube upside down on a clean absorbent paper for 2 minutes to ensure the precipitation in the tube. The mixture of $\mathrm{fg} 500 \mu \mathrm{I}$ buffer and $\mathrm{K} 5 \mu$ I protease was prepared. Add $500 \mu$ I of the prepared mixture, vortex and mix well until the solution has no agglomeration. $65{ }^{\circ} \mathrm{C}$ water bath for 10-30 minutes, during which the mixture was reversed several times. Add $500 \mu \mathrm{I}$ isopropanol and mix thoroughly until there is filamentous or cluster genomic DNA. Centrifugation was performed at 8000 rpm for 8 minutes, and the supernatant was slowly discarded. Place the centrifuge tube upside down on a clean absorbent paper to ensure that it settles in the tube. Add $500 \mu \mathrm{I} 70 \%$ ethanol, vortex oscillation for 5 seconds, centrifugation at $8000 \mathrm{rpm}$ for 3 minutes, and discard the supernatant. Place the centrifuge tube upside down on a clean absorbent paper for 5 minutes to ensure that the sediment is in the tube. Air dry the DNA precipitate until all the liquid evaporates (at least 5 minutes). Add $200 \mu$ I buffer TB, vortex at low speed for 5 seconds, and take a water bath at $65^{\circ} \mathrm{C}$ for 1 hour to dissolve DNA. DNA was quantified by UV spectrophotometer. The OD value was measured at $260 \mathrm{~nm}$ and $280 \mathrm{~nm}$, and the concentration and purity of DNA were calculated. The concentration range of DNA was $0.2 \sim 0.8 \mu \mathrm{g} / \mu \mathrm{L}$, and the od260nm / od280nm was $1.6 \sim 2.0$.

\section{PCR-LDR method to determine genotype}

Take $50 \mu$ of peripheral blood DNA extraction product for genetic polymorphism detection. Select the presence or absence of rs $2242480 * 5 / * 18$, CYP 2 C $9 * 2 / * 13$ CYP2C19 *2 $/ * 3$ from the NCBI dSNP database, and use oligo6.0 software to design and synthesize primers (Table 1). According to the molecular weight of the primers and probes, dilute with TE to $50 \mathrm{pmol} / \mu \mathrm{l}$ as the mother solution; in the case of multiplex PCR and LDR, the mother solutions of the primers are mixed in equal proportions to form a mixture solution that can be directly reacted. Use $3 \%$ agarose gel electrophoresis to detect the effect of the PCR product and determine the amount of it as a template in the LDR reaction.

\section{Table 1. Primer sequences}

\begin{tabular}{|lll|}
\hline Primers & F & R \\
rs2242480 *5 & TGCATTTTCTGCGTGACAGAAG & AGGAACGAAATGATGTGGTTAT \\
rs2242480*18 & AAGTGATTTGGCTGGATTG & AGACCCTCTTCCACCTTCT \\
CYP2C9 *2 & TCAGAGTTTCTGGGGAAGC & GAGGGTAGAGAGGATATCTGATA \\
CYP2C9 *13 & TTGCTCGAGGACAAGTTC & TCTGATTTGGGGACCACAG \\
CYP2C19*2 & AGCTGCACTGTGACAAGCT & CATTCGTCTGTTTCCCATT \\
CYP2C19*3 & CATTTTCTGCTTGACAGAAGA & GGAACAAAATGATGTGGTTAT \\
\hline
\end{tabular}




\section{Statistical methods}

Use indicators such as mean \pm standard deviation or median (interquartile range) to describe numerical variables, and report the frequency and proportion of each classification for categorical variables. Comparison of differences between groups: t-test or rank-sum test is selected for continuous variables according to normality and homogeneity of variance; categorical variables and Hardy-Weinberg balance test Fisher exact probability test. Use Hapview 4.2 software and Phase2.1 software to construct haplotypes; use univariate and multivariate unconditional Logistic regression to analyze the relationship between the genotype distribution of each polymorphic locus and the risk of ATLI, and use odds ratio (OR) And its 95\% confidence interval (Confidence Interval, $\mathrm{Cl}$ ) means that dummy variables are set for multi-categorical variables. All tests are two-sided tests, and the test level is taken as 0.05 , and $P \leq 0.05$ indicates that the difference is statistically significant.

\section{Results}

\section{General situation of the research object}

There are statistical differences between the case group and the control group in terms of gender, age, $\mathrm{BMI}$, weight, the liver function indexes of the case group are significantly higher than those of the control group (Table 2).

\section{Table 2. General comparison of ATLI and control group}




\begin{tabular}{|llll|}
\hline Features & Case & Control & P value \\
\hline Case & $612(27.1)$ & $1643(72.9)$ & \\
\hline Men & $362(59.2)$ & $1049(63.8)$ & 0.040 \\
\hline Age & $73.28(12.92)$ & $67.55(12.51)$ & $<0.001$ \\
\hline Age groups: & & & $<0.001$ \\
\hline$<60$ & $80(13.1)$ & $422(25.7)$ & \\
\hline $60 \sim$ & $131(21.4)$ & $467(28.4)$ & \\
\hline $70 \sim$ & $175(28.6)$ & $436(26.5)$ & \\
\hline $80 \sim$ & $226(36.9)$ & $318(19.4)$ & \\
\hline BMI,means (SD),kg/m ${ }^{2}$ & $24.57(4.56)$ & $24.84(4.27)$ & 0.494 \\
\hline Smoking in past / now & $220(36.1)$ & $693(42.4)$ & 0.506 \\
\hline Drinking in past / now & $64(10.5)$ & $252(15.5)$ & 0.473 \\
\hline AST(U/L) & $1.24(0.76)$ & $1.43(0.93)$ & $<0.001$ \\
\hline ALT(U/L) & $1.09(0.63)$ & $1.09(0.57)$ & 0.026 \\
\hline TBil(U/L) & $2.70(0.98)$ & $2.73(1.00)$ & 0.015 \\
\hline
\end{tabular}

2. The PCR amplification products of the SNPs in rs2242480, CYP2C9 and CYP2C19

$3 \%$ agarose gel electrophoresis detection, observe the effect of the PCR product, and determine the amount of it as a template in the LDR reaction. Figure 1 is the PCR electrophoresis diagram (the main observation in the experiment is whether the PCR is successful, and whether each site is good cannot be observed by electrophoresis): Marker: 100, 200, 300, 400, 500, 6000.

The SNP typing results of ABI 3130XL showed that there were 4 genotypes for each SNPs of rs2242480, CYP2C9 and CYP2C19. T, CT, CC and C for rs2242480, GG, GA, A and G for CYP2C9, GA, AA, G and GG for CYP2C19. DNA sequencing peak chromatograms for these genotyping results were shown in Figure 2 (a, $\mathrm{b}$ and $\mathrm{c}$, respectively).

\section{Hardy-Weinberg balance test}

The Hardy-Weinberg equilibrium test was performed on the polymorphic loci of the RIF gene, as shown in Table 3. It can be seen that the genotype at rs2242480*5/*18 meets the Hardy-Weinberg equilibrium $(P>0.05)$; the genotype at CYP2C9 and CYP2C19 does not reach the Hardy-Weinberg equilibrium ( $P$ $<0.01)$.

Table 3. Hardy-Weinberg test of the genotype distribution of polymorphic loci in the control group 


\begin{tabular}{|llll|}
\hline Polymorphic site & Actual frequency & Theoretical frequency & P value \\
\hline rs2242480 T & $110.54(65.75)$ & $103.28(64.43)$ & 0.019 \\
\hline CT & $137.51(5.80)$ & $138.15(8.97)$ & 0.106 \\
\hline CC & $4.26(1.19)$ & $4.35(1.26)$ & 0.141 \\
\hline C & $9.01(4.22)$ & $7.82(3.06)$ & $<0.001$ \\
\hline CYP2C9 GG & $214.39(83.14)$ & $207.12(72.23)$ & 0.044 \\
\hline GA & $74.67(40.96)$ & $80.35(36.98)$ & 0.002 \\
\hline A & $4940(9194)$ & $28545(5218)$ & $<0.001$ \\
\hline G & $226(36.9)$ & $318(19.4)$ & 0.374 \\
\hline CYP2C19 GA & $24.57(4.56)$ & $24.84(4.27)$ & 0.494 \\
\hline AA & $220(36.1)$ & $693(42.4)$ & $<0.001$ \\
\hline G & $64(10.5)$ & $252(15.5)$ & 0.473 \\
\hline GG & $2.70(0.98)$ & $2.73(1.00)$ & 0.015 \\
\hline
\end{tabular}

3. Genotype distribution of NAT2 polymorphic loci

Table 4 shows the distribution of RIF polymorphism sites in the ATLI group and the control group. It can be seen that the proportion of wild type at the above three RIF polymorphic sites in ATLI group and control group is higher than that of heterozygous type, and heterozygous type is higher than that of mutant homozygous type. Only the different genotypes at CYP2C9 locus are found in ATLI group and control group. The distribution difference between the groups was statistically significant.

\section{Table 4. Genotype distribution of RIF polymorphic loci}

\section{Association between the genotype of RIF polymorphism and ATLI}

The RIF gene is mainly mutated at three sites, namely CYP2C9 *2, CYP2C9*13 and CYP2C19 *2. Univariate and multivariate unconditional Logistic regression were used to analyze the association between different genotypes (additive models) of RIF polymorphism sites and ATLI. Univariate analysis only found that CT genotype at CYP2C9 *2 was significantly associated with ATLI, with an OR value of $1.098(0.879,1.372)$. After adjusting for gender, age, and region, the results of multivariate analysis only found CYP2C9 *2 There is a significant correlation between GA genotype at locus and ATLI, with an OR value of $1.214(0.960,1.535)$ The results are shown in Table 5. For the three SNPs at RIF polymorphism sites, single factor and multivariate unconditional logistic regression were used to analyze the relationship between the genetic model of these three SNPs and ATLI in the dominant model and recessive model. The results are shown in Table 5 . It can be seen that the results of single factor and multivariate analysis found that CYP2C9 *2 has an association with ATLI in the dominant model, and the 


\begin{tabular}{|c|c|c|c|c|c|}
\hline Clinical prognosis & Case & Control & $\mathrm{X}^{2}(95 \% \mathrm{Cl})$ & $P$ value & OR values are 1.098 \\
\hline $\mathrm{rs} 2242480 \mathrm{~T}$ & $133(21.7)$ & $202(12.3)$ & $1.981(1.555,2.523)$ & 0.144 & $1.214(0.960,1.535)$ \\
\hline CT & $156(25.6)$ & $260(15.8)$ & $1.820(1.453,2.279)$ & 0.245 & \multirow{5}{*}{$\begin{array}{l}\text { No correlation } \\
\text { between genetic } \\
\text { models at other sites } \\
\text { and ATLI was found to } \\
\text { be statistically } \\
\text { significant. }\end{array}$} \\
\hline $\mathrm{CC}$ & 75 (12.3) & $128(7.8)$ & $1.653(1.223,2.235)$ & 0.040 & \\
\hline C & $139(22.7)$ & 303 (18.4) & $1.300(1.036,1.630)$ & 0.073 & \\
\hline CYP2C9 GG & $142(23.2)$ & $321(19.5)$ & $1.244(0.995,1.556)$ & $<0.001$ & \\
\hline GA & $155(25.3)$ & $358(21.8)$ & $1.217(0.980,1.512)$ & $<0.001$ & \\
\hline A & $199(32.5)$ & $347(21.1)$ & $1.800(1.464,2.212)$ & $<0.001$ & \multirow{6}{*}{$\begin{array}{l}\text { Table } 5 \text {. The } \\
\text { genotypes of RIF } \\
\text { polymorphism loci, the } \\
\text { association between } \\
\text { different genetic } \\
\text { models and ATLI }\end{array}$} \\
\hline G & $156(25.6)$ & $260(15.8)$ & $1.820(1.453,2.279)$ & $<0.001$ & \\
\hline CYP2C19 GA & $64(10.5)$ & $94(5.7)$ & $1.925(1.380,2.683)$ & 0.053 & \\
\hline$A A$ & $99(16.2)$ & $167(10.2)$ & $1.706(1.304,2.230)$ & 0.481 & \\
\hline G & $116(19.0)$ & $215(13.1)$ & $1.553(1.212,1.990)$ & 0.653 & \\
\hline GG & $199(32.5)$ & $347(21.1)$ & $1.800(1.464,2.212)$ & 0.287 & \\
\hline
\end{tabular}

\begin{tabular}{|ll|}
\hline Polymorphic site & OR \\
\hline rs2242480 T & $1.609(1.082,2.391)$ \\
\hline CT & $1.454(1.067,1.982)$ \\
\hline CC & $1.139(0.902,1.438)$ \\
\hline C & $1.111(0.883,1.399)$ \\
\hline CYP2C9 CT & $1.098(0.879,1.372)$ \\
\hline GA & $1.214(0.960,1.535)$ \\
\hline A & $1.475(0.952,2.284)$ \\
\hline G & $1.540(1.094,2.168)$ \\
\hline CYP2C19 GA & $1.394(1.057,1.838)$ \\
\hline AA & $1.264(0.978,1.633)$ \\
\hline G & $1.609(1.082,2.391)$ \\
\hline GG & $1.454(1.067,1.982)$ \\
\hline
\end{tabular}

\section{Discussion}


In the study of genetic epidemiology, especially in the study of association analysis for finding and locating disease-causing genes, haplotype, as a relatively common data type, is playing an important role[9]. A single SNP site provides very limited information for complex diseases, and complex diseases are often caused by the interaction of DNA mutations at multiple sites. Many literature reports that haplotypes contain more Linkage Disequilibrium (LD) information, which is conducive to finding and locating pathogenic sites in association analysis. Therefore, studies based on haplotypes are better than studies based on single SNPs. The research can bring greater effect[10]. RIF is a macromolecular drug, which is mainly excreted via bile, and its own liver toxicity is relatively low. The main metabolic pathway of RIF is deacetylation to produce DesacetyIRifampicin, which is then hydrolyzed to produce 3-Formyl Rifampicin, most of which are secreted into bile through the bile duct, excreted into the small intestine, and partly enters Enterohepatic circulation, and finally excreted with 25-desacetyl rifampicin. Studies have shown that the metabolites of RIF have no obvious hepatotoxicity[11]. The liver damage caused by RIF is mainly hepatic and cholestatic mixed hepatitis changes, which increase the serum bilirubin level and transaminase level. RIF interferes with the binding of bilirubin and glucuronic acid in liver cells, and competes with bilirubin for excretion, increasing the unbound and bound bilirubin in the blood, causing cholestatic liver damage, and the degree of damage Related to dose[12]. At the same time, RIF also has the effect of inducing a variety of metabolic enzymes in the liver [13], aggravating the toxicity of combined drugs. When combined with INH, not only the incidence of liver damage increases, but also the degree[14]. In addition, RIF can cause type IV hypersensitivity and cause liver damage. Studies have found that, as an inducer of cytochrome P450, RIF can increase the toxic metabolites of INH and significantly aggravate the liver damage of $\mathrm{INH}$.

\section{References}

1. Bryant KE, Yuan Y, Engle M, Kurbatova EV, Allen-Blige C, Batra K, Brown NE, Chiu KW, Davis H, Elskamp M, Fagley M, Fedrick P, Hedges KNC, Narunsky K, Nassali J, Phan M, Phan H, Purfield AE, Ricaldi JN, Robergeau-Hunt K, Whitworth WC, Sizemore EE, \%J Contemporary clinical trials. Central monitoring in a randomized, open-label, controlled phase 3 clinical trial for a treatment-shortening regimen for pulmonary tuberculosis. 2021: 106355.

2. Ma J, Huang M, Wang SH, Tan QM, Zhang LS \%J Explore. Undiagnosed pleural effusion treated with traditional Chinese medicine: A case report. 2021.

3. Reckers A, Huo S, Esmail A, Dheda K, Bacchetti P, Gandhi M, Metcalfe J, Gerona R \% J Journal of chromatography. B, Analytical technologies in the biomedical, sciences life. Development and validation of a liquid chromatography-tandem mass spectrometry method for quantifying delamanid and its metabolite in small hair samples. 2020, 1169: 122467.

4. Verma D, Chan ED, Ordway DJ \%J Immunological reviews. The double-edged sword of Tregs in M tuberculosis, M avium, and M absessus infection. 2021. 
5. Zugazaga A, Stachno MA, García A, Tovar G, Benito V, Guasch I, Nogueira I, Puyalto P, Sampere J $\% J$ European radiology. Pulmonary artery pseudoaneurysms: endovascular management after adequate imaging diagnosis. 2021.

6. Anjani QK, Permana AD, Cárcamo-Martínez Á, Domínguez-Robles J, Tekko IA, Larrañeta E, Vora LK, Ramadon D, Donnelly RF \%J European journal of pharmaceutics, e.V biopharmaceutics : official journal of Arbeitsgemeinschaft fur Pharmazeutische Verfahrenstechnik. Versatility of hydrogel-forming microneedles in in vitro transdermal delivery of tuberculosis drugs. 2021, 158: 294-312.

7. Sanjay S, Girish C, Toi PC, Bobby Z \%J Canadian journal of physiology, pharmacology. in vivoQuercetin Modulates Nrf2 and NF-KB/TLR-4 Pathways to Protect against Isoniazid and Rifampicin Induced Hepatotoxicity. 2021.

8. Shehu Al, Zhu J, Li J, Lu J, McMahon D, Xie W, Gonzalez FJ, Ma X \%J Toxicological sciences : an official journal of the Society of Toxicology. Targeting xenobiotic nuclear receptors PXR and CAR to prevent cobicistat hepatotoxicity. 2021.

9. $\quad$ Su Q, Kuang W, Hao W, Liang J, Wu L, Tang C, Wang Y, Liu T \% J Mediators of inflammation. Antituberculosis Drugs (Rifampicin and Isoniazid) Induce Liver Injury by Regulating NLRP3 Inflammasomes. 2021, 2021: 8086253.

10. Ware BR, Liu JS, Monckton CP, Ballinger KR, Khetani SR \%J Toxicological sciences : an official journal of the Society of Toxicology. Micropatterned Coculture with 3T3-J2 Fibroblasts Enhances Hepatic Functions and Drug Screening Utility of HepaRG Cells. 2021.

11. Hegarty CE, lanni AM, Kohn PD, Kolachana B, Gregory M, Masdeu JC, Eisenberg DP, Berman KF $\% J$ Biological psychiatry. Cognitive neuroscience, neuroimaging. Polymorphism in the ZNF804A Gene and Variation in D and D/D Dopamine Receptor Availability in the Healthy Human Brain: A Dual Positron Emission Tomography Study. 2021.

12. Kholina A, Kozyrenko M, Artyukova E, Sandanov D, Selyutina I \%J Genetica. Genetic diversity of Oxytropis section Xerobia (Fabaceae) in one of the centres of speciation. 2021.

13. Medina-Montes A, Carrillo-Gonzalez DF, Hernández-Herrea DY \%J Tropical animal health, production. Association of a genetic polymorphism in the BMPR-1B gene, and non-genetic factors with the natural prolificacy of the Colombian-haired sheep. 2021, 53(2): 206.

14. Reia TA, Silva RFD, Jacomini AM, Moreno AMG, Ferezin LP, Pereira SC, Lacchini R, Dionísio TJ, Santos CF, Zago AS \%J Journal of physical activity, health. Acute Exercise, Plasma Nitric Oxide, and Blood Pressure in Older Adults With Different Levels of Training Status: The Influence of Polymorphisms of Endothelial Nitric Oxide Synthase. 2021: 1-8.

\section{Declarations}




\section{Ethics approval and consent to participate}

This research was approved by the IRB of The First Affiliated Hospital of Chongqing Medical University in China.

Consent for publication: All authors agree to publish this research.

\section{Availability of data and materials}

The datasets generated and/or analyzed during the current study are not publicly available due to the confidentiality policy of patients but are available from the corresponding author on reasonable request.

Conflict of Interest Disclosures: No authors declare a potential conflict of interest.

Funding: Scientific Research Projects on Basic Scientific and Technological Strategic Cooperation of Nanchong Municipal Schools (No. 18SXHZ0362); Scientific Research Projects of Sichuan Education Department (No. 18ZB0221);Sichuan grass-roots health development research center project(No.SWFZ18-Z10)

\section{Author Contributions}

Author Contributions: Pan and Su had full access to all the data in the study and take responsibility for the integrity of the data and the accuracy of the data analysis.

Study concept and design: Pan and Su.

Acquisition, analysis, or interpretation of data: Pan and Su.

Drafting of the manuscript: Pan and Su.

Critical revision of the manuscript for important intellectual content: All authors.

Statistical analysis: Pan and Su.

Administrative, technical, or material support: Pan.

Study supervision: Pan.

\section{Figures}




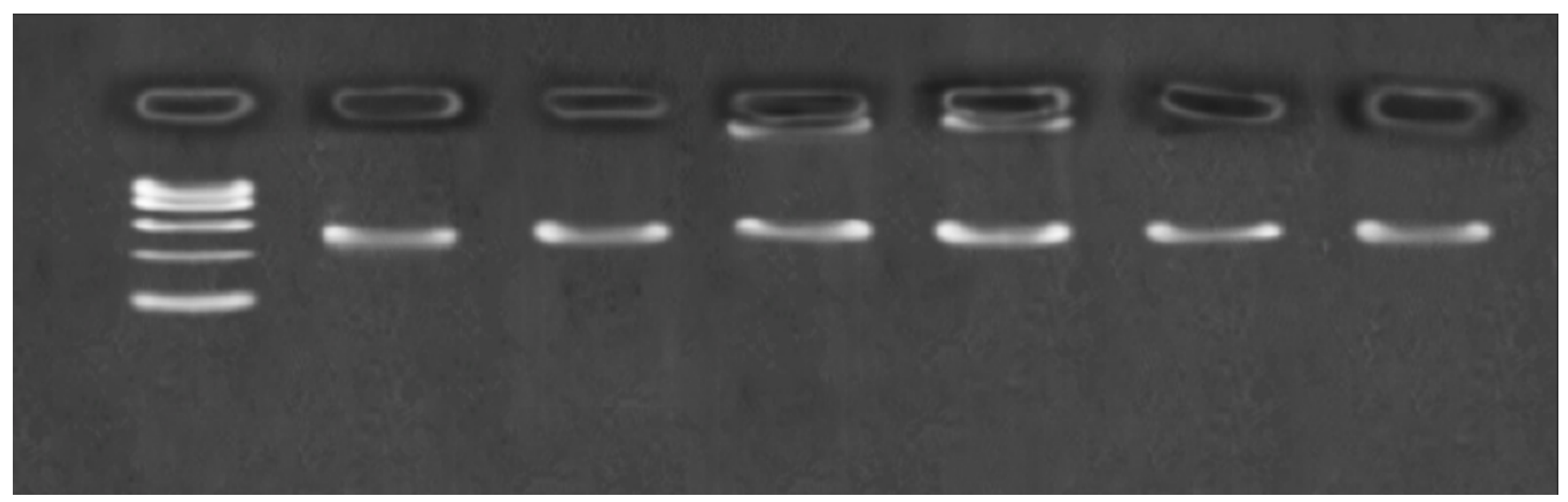

Figure 1

Detection of SNP PCR product in agarose gel electrophoresis

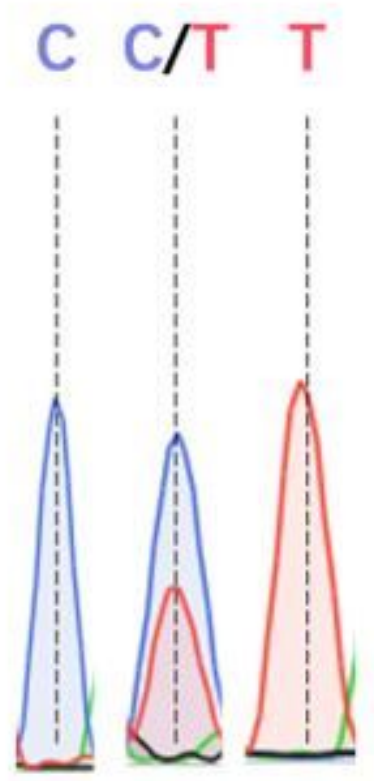

a

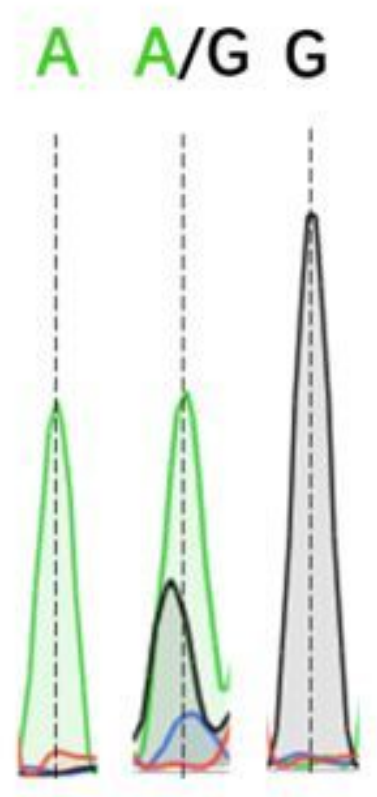

b
A $A / G$ G

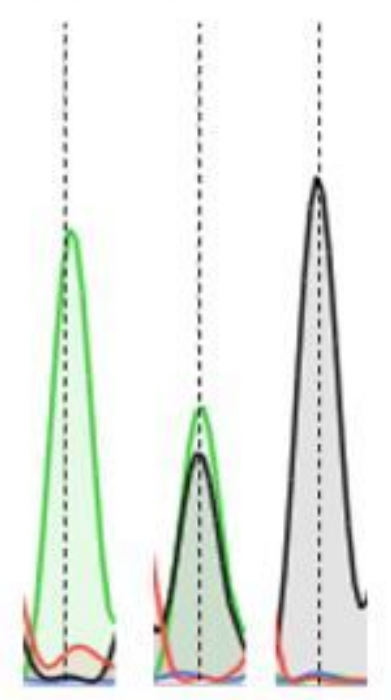

C

\section{Figure 2}

DNA sequencing peak chromatograms for the rs2242480, CYP2C9 and CYP2C19 genotyping, dotted line indicates the mutation site: (a) rs2242480 polymorphism, (b) CYP2C9 polymorphism, (c) CYP2C19 polymorphism. 